\title{
Subcutaneous Infusion of Pamidronate in a Hospice Patient with Hypercalcemia: A Case Report
}

\author{
Chris Vandevelde and Jordan Ho
}

Can J Hosp Pharm. 2021;74(1):80-2

\section{INTRODUCTION}

Hypercalcemia is a complication of cancer, reported to affect between $10 \%$ and $40 \%$ of patients with cancer and occurring in those with both solid tumours and hematologic malignancies. ${ }^{1-4}$ Cancer is the most common cause of hypercalcemia in the inpatient setting, most frequently breast, renal, and lung cancer and multiple myeloma. ${ }^{1,2,5}$ Malignancy is usually clinically evident by the time it causes hypercalcemia, and patients with hypercalcemia of malignancy often have a poor prognosis. ${ }^{1}$

There are several mechanisms by which hypercalcemia of malignancy can occur. The major mechanism, associated with approximately $80 \%$ of cases, is secretion of parathyroid hormone-related protein by the tumour. ${ }^{1-4}$ Other mechanisms include local release of cytokines (including osteoclastactivating factors) from osteolytic metastatic lesions, tumour production of 1,25-dihydroxyvitamin $\mathrm{D}$ (calcitriol), and production or secretion of parathyroid hormone secondary to parathyroid carcinoma. ${ }^{1,2,4}$

For hypercalcemia and bone pain, IV administration of bisphosphonates is a recognized, first-line treatment option. ${ }^{2-4}$ In our health authority, Fraser Health (located in the Lower Mainland of British Columbia), IV pamidronate is one of the first-line bisphosphonates in the treatment of hypercalcemia. However, for patients receiving palliative care in hospice settings, Fraser Health policy stipulates that IV administration is not an option because hospice nursing staff are not trained to provide the higher level of monitoring required with this route of administration. Additionally, other risks and barriers to IV administration in the palliative care population have been reported, including thrombophlebitis, pain secondary to needle insertion, difficult venous access, and infection. ${ }^{6,7}$

The subcutaneous (SC) administration of bisphosphonates for patients receiving palliative care has been reported as an alternative to IV administration. ${ }^{6-8}$ To determine the feasibility of an alternative route of administration in our setting, we searched PubMed and Ovid MEDLINE using the terms "hypercalcemia", "bisphosphonates", and/or "injections, subcutaneous". This search identified the same 3 reports of SC administration of bisphosphonates in the palliative setting of which we were already aware ${ }^{6-8}$; no additional reports were found. Clodronate, a first-generation bisphosphonate, has been used in the palliative care setting in Edmonton, Alberta, with reported safety and efficacy. ${ }^{7,8}$ Duncan ${ }^{6}$ reported the SC use of pamidronate in a UK hospital, where the drug was administered to 10 patients, of whom 7 had a biochemical response, with serum calcium decreasing to within normal limits. However, although there is reported evidence for SC administration of clodronate, this drug is unavailable for use in our health authority, and although Duncan ${ }^{6}$ reported biochemical response in 7 of 10 patients who received SC pamidronate, she did not discuss patients' symptoms or their symptomatic response. As such, evidence for and timing of symptomatic efficacy to guide SC administration of pamidronate is lacking from the literature at this time.

We report a case of hypercalcemia of malignancy in a patient who experienced biochemical and symptomatic response to pamidronate administered by the SC route.

\section{CASE REPORT}

A 70-year-old man with fungating inoperable penile carcinoma with lung and lymph node metastases did not wish to undergo further investigation or oncologic treatment.* The patient was transferred from an inpatient palliative care unit to hospice on August 31, 2018. He had a history of malignancy-related hypercalcemia, which had responded to IV administration of pamidronate on August 12, 2018 (before transfer to hospice). This was the first and only dose of pamidronate that the patient received, and there were

*The patient died prior to consent for publication being obtained, and repeated attempts to contact the substitute decision-maker were unsuccessful. 
both biochemical (Table 1) and symptomatic (increased energy, decreased nausea and confusion) responses.

During hospice team rounds on September 12, it was reported that the patient was more confused than previously noted during this admission, being unable to follow simple directions. The patient was not oriented to place or time, was not eating or drinking, and was refusing his oral medications and routine care. A urine sample was sent for culture and sensitivity testing, which yielded no growth. No blood was drawn for culture, as per the patient's goals of care; the patient was afebrile. IV fluids were not initiated, as per hospice policy. There were no recent medication changes thought to be contributory to the patient's change in function, and the patient was not receiving calcium or vitamin D supplements. Routine blood tests were ordered, including serum calcium, albumin, and creatinine; the results of previous liver and kidney function tests at the inpatient palliative unit were within normal limits. Laboratory results on September 14 revealed that the patient had elevated serum calcium (Table 1).

Pamidronate $90 \mathrm{mg}$ in $500 \mathrm{~mL}$ of $0.9 \%$ sodium chloride (normal saline) via SC infusion was started on September 18; the drug was infused over $24 \mathrm{~h}$ via gravity drip, similar to how hypodermoclysis is administered in our hospice units. Follow-up blood tests on September 26 showed a reduction of serum calcium to within the normal range (but no corresponding measurement of albumin was ordered at that time; see Table 1). In addition, the patient's symptoms resolved (return of appetite; alert and oriented to person, place, and time; and taking oral medications) within $24 \mathrm{~h}$ of completing the infusion. Notably, for the patient's comfort during pamidronate administration, the infusion site was re-located from the upper arm to the abdomen.

\section{DISCUSSION}

Although there is substantial evidence supporting the use of IV bisphosphonates as first-line therapy for hypercalcemia of malignancy, there is limited literature showing evidence for the safety and efficacy of bisphosphonate administration via the $\mathrm{SC}$ route and nothing describing the kinetics of SC bisphosphonate. The most robust evidence for SC administration of bisphosphonate was detailed in a retrospective cohort study, in which Roemer-Bécuwe and others ${ }^{7}$ reviewed the use of SC clodronate for management of hypercalcemia and/or bone pain in the palliative care population in Edmonton. ${ }^{7}$ The 149 patients in that study received a total of 254 infusions over a 4-year period and were evaluated retrospectively for safety and efficacy of SC administration of clodronate. The reported toxic effects included pain $(7.9 \%$ of infusions), swelling (3.1\%), bruising (2.8\%), redness (6\%), and discharge $(0.4 \%)$. The authors reported that local toxicity was mild, with discomfort being resolved by application of hot packs and discontinuation of the infusion, the latter being required for only 2 infusions (1.0\%). Of the 90 infusions administered for hypercalcemia management, only 43 met the criteria for evaluation of efficacy (because of missing data), with 32 (74.4\%) achieving normalization within 5 days, $3(7.0 \%)$ during week 2 after the infusion, and 8 $(18.6 \%)$ having no decrease in calcium. The authors reported a significant overall decrease in calcium levels $(p<0.0001)$ within 5 days after SC clodronate infusion. Although this cohort study provided evidence for the safety and efficacy of clodronate for management of hypercalcemia of malignancy, ${ }^{7}$ this drug is not available for use in Fraser Health and could not be considered as an option for management in our patient. However, the same study was considered to provide evidence for use of bisphosphonate therapy through the SC route of administration in the palliative care population, which provided a rationale to consider use of another drug, pamidronate, via the $\mathrm{SC}$ route.

In a case series report, Duncan ${ }^{6}$ described the SC administration of pamidronate to 10 patients; for each patient $90 \mathrm{mg}$ of drug was diluted in 375 to $1000 \mathrm{~mL}$ of normal saline and administered via an SC butterfly needle over 4 to $24 \mathrm{~h}$. All of the patients had cancer at one of the

TABLE 1. Laboratory Results for Hospice Patient with Hypercalcemia ${ }^{a}$

\begin{tabular}{lcccc} 
Date & $\begin{array}{c}\text { Serum Calcium } \\
(\mathbf{m m o l} / \mathbf{L})\end{array}$ & $\begin{array}{c}\text { Albumin } \\
(\mathbf{g} / \mathbf{L})\end{array}$ & $\begin{array}{c}\text { Ionized Calcium } \\
(\mathbf{m m o l} / \mathbf{L})\end{array}$ & $\begin{array}{c}\text { Corrected Calcium } \\
(\mathbf{m m o l} / \mathbf{L})\end{array}$ \\
\hline Normal range & $2.1-2.6$ & $35-50$ & $1.15-1.32$ & NA \\
\hline August 11 & Not ordered & 21 & 1.79 & NA \\
\hline August 14 & Not ordered & Not ordered & 1.49 & NA \\
\hline August 17 & 1.99 & 17 & Not ordered & 2.57 \\
\hline September 14 & 3.48 & 29 & 1.91 & 3.7 \\
\hline September 26 & 2.18 & Not ordered & Not ordered & NA \\
\hline
\end{tabular}

NA = not applicable/not available.

aPamidronate was administered by the IV route on August 12 in the inpatient palliative care unit. The patient was transferred to hospice care on August 31 and received pamidronate by the SC route on September 18 . 
following sites: breast $(n=4)$, pancreas $(n=1)$, lung $(n=1)$, myeloma $(n=1)$, prostate $(n=1)$, and unknown primary origin $(n=2)$. Biochemical response, defined as reduction of serum calcium to within normal limits, occurred in 7 of the 10 patients. Inflammation of the SC sites was the most common adverse event and appeared more likely with quicker infusions, over 4 to $5 \mathrm{~h}$; inflammation was less pronounced with longer infusions, over 12 to $24 \mathrm{~h}$. Only 1 patient did not tolerate the infusion because of painful stinging. Unfortunately, there was no description of patients' symptomatic responses in this case series.

Denosumab is another SC treatment option for hypercalcemia of malignancy ${ }^{9}$; however, this drug was not considered in our case because the contracted pharmacy did not routinely stock it, and the acquisition delay ( $>24 \mathrm{~h}$ ) was thought to be unacceptable. In addition, the cost (approximately $\$ 600$ ) would be covered by the BC Cancer Agency only if the patient were registered with the agency; the patient in our case was not registered (based on his goals of care and his desire not to receive any cancer-specific treatment), and the pharmacy therefore had no way of recouping its costs. The prescriber had previously used SC pamidronate for another patient; on the basis of this experience, combined with the reasons outlined above, we proceeded with this treatment option.

This case and previous cases reported in the literature indicate that SC pamidronate infusion is both well tolerated and worthwhile in the palliative care setting. Greater-thanusual dilution and a slower infusion rate add to the tolerability of administration of the drug by the SC route and represent a reasonable option for the treatment of hypercalcemia in Fraser Health hospices. We recommend that pamidronate $90 \mathrm{mg}$ be diluted in $500 \mathrm{~mL}$ of normal saline and administered via SC infusion over $24 \mathrm{~h}$ (similar to how we administer hypodermoclysis in our hospice units), as the larger volume of fluid is also of benefit for patients with hypercalcemia. Patients should be monitored for infusionsite reactions (e.g., redness), and the SC infusion is likely best sited in the abdomen (as per the prescriber's previous experience with hypodermoclysis) where the infusion volume might be best tolerated; other potential sites include the thigh or the chest. The patient described here experienced only mild discomfort at the initial injection site, and this pain resolved once the infusion was re-sited to the abdomen.

\section{CONCLUSION}

In this case, pamidronate SC was safe and efficacious for treatment of the patient's hypercalcemia of malignancy. This case furthers our knowledge of how best to administer pamidronate by this route, with regard to fluid volume, rate of infusion, and infusion site.

\section{References}

1. Steward AF. Clinical practice. Hypercalcemia associated with cancer N Engl J Med. 2005;352(4):373-9.

2. Goldner W. Cancer-related hypercalcemia. J Oncol Pract. 2016;12(5): 426-32.

3. Kovacs CS, MacDonald SM, Chik CL, Bruera E. Hypercalcemia of malignancy in the palliative care patient: a treatment strategy. J Pain Symptom Manage. 1995;10(3):224-32.

4. Mirrakhimov AE. Hypercalcemia of malignancy: an update on pathogenesis and management. North Am J Med Sci. 2015;7(11):483-93.

5. Gastanaga VM, Schwartzberg LS, Jain RK, Pirolli M, Quach D, Quigley JM, et al. Prevalence of hypercalcemia among cancer patients in the United States. Cancer Med. 2016;5(8):2091-100.

6. Duncan AR. The use of subcutaneous pamidronate. J Pain Symptom Manage. 2003;26(1):592-93.

7. Roemer-Bécuwe C, Vigano A, Romano F, Neumann C, Hanson J, Quan $\mathrm{HK}$, et al. Safety of subcutaneous clodronate and efficacy in hypercalcemia of malignancy: a novel route of administration. J Pain Symptom Manage. 2003;26(3):843-48.

8. Walker P, Watanabe S, Lawlor P, Hanson J, Pereira J, Bruera E. Subcutaneous clodronate: a study evaluating efficacy in hypercalcemia of malignancy and local toxicity. Ann Oncol. 1997;8(9):915-6.

9. Denosumab [monograph]. In: Badry N, editor. Cancer drug manual. Provincial Health Services Authority, BC Cancer Agency; 2019 [cited 2019 Jan 25]. Available from: www.bccancer.bc.ca/health-professionals/ clinical-resources/cancer-drug-manual

Chris Vandevelde, BSCPharm, ACPR, is a Clinical Pharmacy Specialist in the Palliative Care Program (Fraser East), Fraser Health Authority, Abbotsford, British Columbia.

Jordan Ho, BScPharm, is a Clinical Pharmacist with the Fraser Health Authority, Surrey, British Columbia.

Competing interests: None declared.

Address correspondence to:

Chris Vandevelde

Pharmacy Community Programs

Fraser Health

32780 Marshall Road

Abbotsford BC V2S 1J7

email: Chris.Vandevelde@fraserhealth.ca

Funding: None received. 